\title{
Consolidation by Prefabricated Vertical Drains with a Threshold Gradient
}

\author{
Xiao Guo, ${ }^{1}$ Kang-He Xie, ${ }^{1}$ and Yue-Bao Deng ${ }^{2}$ \\ ${ }^{1}$ Institute of Geotechnical Engineering, Zhejiang University, Hangzhou 310058, China \\ ${ }^{2}$ Faculty of Architectural Civil Engineering and Environment, Ningbo University, Ningbo 315211, China
}

Correspondence should be addressed to Xiao Guo; 52968791@qq.com

Received 6 August 2014; Accepted 1 December 2014; Published 16 December 2014

Academic Editor: Yuming Qin

Copyright (c) 2014 Xiao Guo et al. This is an open access article distributed under the Creative Commons Attribution License, which permits unrestricted use, distribution, and reproduction in any medium, provided the original work is properly cited.

This paper shows the development of an approximate analytical solution of radial consolidation by prefabricated vertical drains with a threshold gradient. To understand the effect of the threshold gradient on consolidation, a parametric analysis was performed using the present solution. The applicability of the present solution was demonstrated in two cases, wherein the comparisons with Hansbo's results and observed data were conducted. It was found that (1) the flow with the threshold gradient would not occur instantaneously throughout the whole unit cell. Rather, it gradually occurs from the vertical drain to the outside; (2) the moving boundary would never reach the outer radius of influence if $R+1<n$, whereas it will reach the outer radius of influence at some time; (3) the excess pore pressure will not be dissipated completely, but it will maintain a long-term stable value at the end of consolidation; (4) the larger the threshold gradient is, the greater the long-term excess pore pressure will be; and (5) the present solution could predict the consolidation behavior in soft clay better than previous methods.

\section{Introduction}

Preloading with sand or prefabricated vertical drains has been successfully applied to accelerate the consolidation of compressible fine-grained soil deposits for decades. Many authors have developed the consolidation theory for the design of a vertical drain [1-10]. Among these theories, the analytic solutions developed by Barron [1] and Hansbo [3] remain popular and widely used because of their simplicity and ease of use.

All of the above-mentioned solutions assume that Darcy's law is valid. However, several researchers have provided laboratory evidence that Darcy's law should be corrected for the effect of the threshold gradient in some fine-grained soil. Miller and Low [11] found that the threshold gradient is the hydraulic gradient where no flow occurs. This seems to be supported by experimental observations reported by Byerlee [12]. Hansbo [13, 14] proposed a non-Darcian flow law, which implied that the presence of a threshold gradient value, when the gradient is less than this value, may not cease but may dramatically decrease the flow rate. On the other hand, there are several studies that refute this conclusion [15-18].
For example, having conducted permeability tests on four natural clays, Tavenas et al. [18] concluded that Darcy's law was valid in soft clays for hydraulic gradients ranging from 0.1 to 50 . However, it is nearly impossible to verify the validity of Darcy's law with very small gradients.

Although there are some inconsistencies among these studies, it is helpful to examine and assess the impact on the consolidation process due to the existence of the threshold gradient, which was demonstrated by previous experiments. In this study, the flow law of the threshold gradient is assumed to be

$$
v= \begin{cases}0 & |i| \leq i_{0} \\ k\left(|i|-i_{0}\right) & |i|>i_{0}\end{cases}
$$

in which $k$ is the coefficient of permeability, $i$ is the hydraulic gradient, and $i_{0}$ is the threshold gradient. When $i_{0}=0$, (1) degenerates to Darcy's law.

Following equation (1), Pascal et al. [19] obtained a numerical solution using the finite difference method to arrive at approximate analytical solutions for one-dimensional consolidation. Xie et al. [20] presented a general approximate 
analytical solution for one-dimensional consolidation under a time-dependent loading condition and further explored the movement of the seepage boundary in a similar way to (1). Thus, (1) provides an excellent approximation of the effective flow rate. Importantly, it may be simpler to depict the characteristics of the consolidation problem with a nonDarcy flow by using (1).

Various attempts have characterized the influence of the threshold gradient for one-dimension consolidation in clay. However, limited research has focused on the simulation of consolidation behavior using vertical drains that consider the threshold gradient. Hansbo [3] made some approximations and deduced a simple closed-form solution for radial consolidation using vertical drains assuming that Darcy's law is valid. Following the approach of Hansbo [3], a general analytical solution with a threshold gradient for radial consolidation by vertical drain is developed in this paper. Afterwards, the influence of the threshold gradient on the consolidation process is investigated.

\section{Mathematical Model}

2.1. Basic Assumptions. Figure 1 presents the schematic representation of an axisymmetric unit cell with the presence of a vertical drain in the center. The vertical drain completely penetrates the soft soil layer, and the top of the layer is freely draining but the bottom is completely impermeable. Thus, the length of the drain well $l$ is equal to the thickness of the soft layer H. $r_{w}$ is the equivalent radius of the drainage well; $r_{e}$ is the radius of the influence zone, which is the radius of the soil cylinder that is dewatered by a drain. $r_{t}$ is the outer radius of the seepage zone. A widespread surcharge loading of $q_{0}$ is simulated by the instantaneous application to the upper boundary and this was kept constant during the consolidation process. Additionally, $r$ and $z$ in Figure 1 indicate the radial coordinates and the depth, respectively.

The assumptions of this paper, some similar to those previously presented by Barron [1] and Hansbo [3], are listed as follows.

(1) Radial and vertical flow can be considered separately.

(2) The quantity of water flowing through the disturbed soil zone into the drain well is equal to the quantity of water flowing out of the drain.

(3) The radial flow in the well can be ignored.

(4) Uniform loading is instantaneously imposed and it is kept constant during the entire consolidation process.

(5) All vertical loads are initially carried by the excess pore water pressure.

(6) The flow in clay obeys (1), while the flow in the vertical drains obeys Darcy's law.

(7) All compressive strains within the soil mass occur in a vertical direction and the equal strain condition is valid.

(8) The smear effect is ignored.

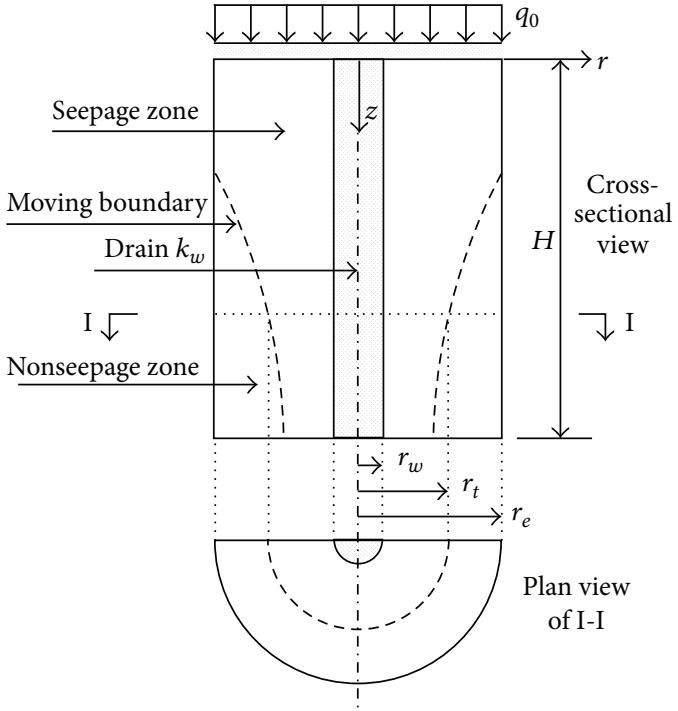

FIgURE 1: The cylindrical unit cell for the vertical drain ground.

2.2. Basic Equation. According to assumption (1), the radial and vertical flow can be considered separately. Thus, as shown in Figure 2, to satisfy the continuity condition for incompressible flow in the radial direction ( $r$-direction in Figure 2), the rate of the soil element's volume change, considering that the radial flow must exclusively be equal to the net flow rate in the radial direction, yields

$$
\frac{\partial V}{\partial t}=\Delta q=\frac{\partial q_{r}}{\partial r} d r
$$

where $q_{r}$ is the net rates of flow at the radial direction. $q_{r}$ can be expressed as

$$
q_{r}=v_{r} r d \theta d z
$$

in which $v_{r}$ is the flow velocity at the radial direction. The rate of volume change of the soil element, considering the radial flow exclusively, can be expressed as

$$
\frac{\partial V}{\partial t}=-\frac{\partial \varepsilon_{v}}{\partial t} r d r d \theta d z
$$

in which $\varepsilon_{v}$ is the volumetric strain of the soil element if only the radial flow is considered.

Substituting (3) and (4) into (2), the separate, general continuity equation for the radial flow under the cylindrical coordinates system can be presented as

$$
\frac{\partial \varepsilon_{v}}{\partial t}+\frac{\partial v_{r}}{\partial r}+\frac{v_{r}}{r}=0
$$

In this paper, the flow of pore water in the soil described by (1) will be incorporated in this study. By expressing the hydraulic gradient, $i$, in terms of the radial excess pore pressure, $u_{r}$, and the unit weight of water, $\gamma_{w}, v_{r}$ can be written as

$$
\begin{gathered}
0 \quad i<i_{0} \\
v_{r}=\frac{k_{h}}{\gamma_{w}} \frac{\partial u_{r}}{\partial r}-k_{h} i_{0} \quad i \geq i_{0} .
\end{gathered}
$$




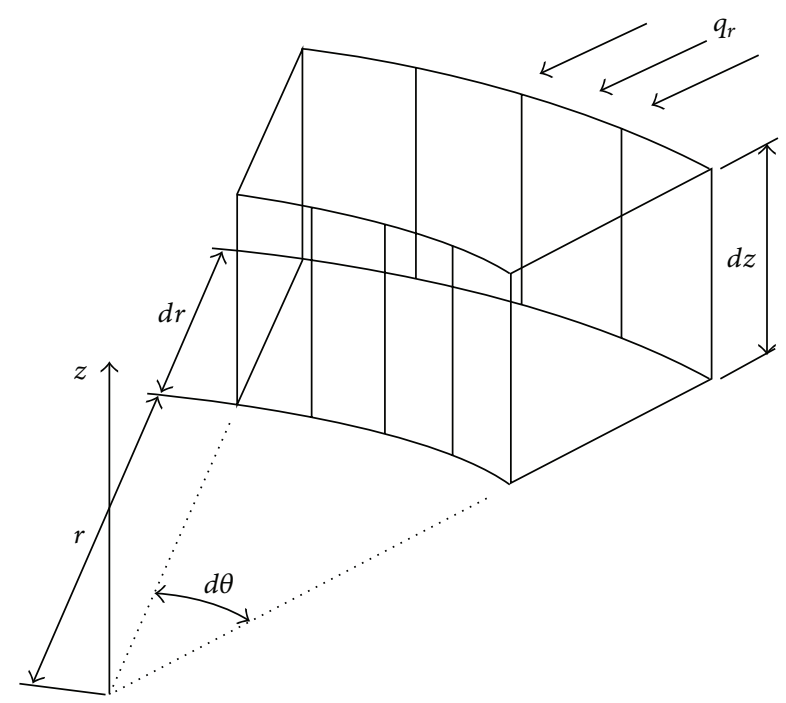

Figure 2: Consolidating the soil element around a vertical drain in a cylindrical system.

Substituting (6) into (5), the inquiry consequently becomes a moving boundary problem as illustrated in Figure 1 and it is governed by the equations that follow:

$$
\begin{gathered}
\frac{k_{h}}{\gamma_{w}} \frac{1}{r} \frac{\partial}{\partial r}\left(r \frac{\partial u_{r}}{\partial r}\right)-\frac{k_{h}}{r} i_{0}=-\frac{\partial \varepsilon_{v}}{\partial t} \quad r_{w} \leq r \leq r_{t} \\
u_{r}=u_{0} \quad r_{t} \leq r \leq r_{e},
\end{gathered}
$$

in which $u_{0}$ is the initial excess pore pressure and is often assumed to be $u_{0}=q_{0} ; r_{t}$ is the outer radius of the seepage zone and the radius of the moving boundary out of which the excess pore pressure is equal to the value of $u_{0}$.

The rate of volumetric strain change of the soil element in the sweep zone depends on the outer radius of the seepage zone, $r_{t}$, and eventually relays the threshold gradient, $i_{0}$. It can be written as follows:

$$
\frac{\partial \varepsilon_{v}}{\partial t}=-m_{v} \frac{\partial \overline{u_{r t}}}{\partial t} \quad r_{w} \leq r \leq r_{t}
$$

where $m_{v}$ is the coefficient of the volume compressibility and $\overline{u_{r t}}$ is the average pore pressure of the seepage zone at any depth. The average pore pressure of the seepage zone can be obtained by

$$
\overline{u_{r t}}=\frac{1}{\pi\left(r_{t}^{2}-r_{w}^{2}\right)} \int_{r_{w}}^{r_{t}} 2 \pi r u_{r} d r \quad r_{w} \leq r \leq r_{t}
$$

in which $u_{r}$ is the excess pore water pressure in the radial direction.

The consolidation equation for the continuity condition of the drain well can be derived using assumptions (3) and (4). Imagining an elementary column of vertical drain with a height of $d z$ as illustrated in Figure 3, the vertical flow through the elementary column can be written as

$$
q_{1}=\frac{k_{w} \pi r_{w}^{2}}{\gamma_{w}} \frac{\partial u_{w}}{\partial z} d t
$$



FIGURE 3: Schematic diagram of the flow rate continuation in a vertical drain.

The radial inflow entering into the elementary column through its outside face in a unit time $d t$ is

$$
q_{2}=2 \pi r_{w}\left(\left.\frac{k_{h}}{\gamma_{w}} \frac{\partial u_{r}}{\partial r}\right|_{r=r_{w}}-k_{h} i_{0}\right) d z d t .
$$

According to assumption (3), the continuity equation in the elementary column of the vertical drain yields

$$
q_{1}+\frac{\partial q_{1}}{\partial z} d z+q_{2}=q_{1}
$$

Substituting (10) and (11) into (12), the following expression is obtained:

$$
\left.\frac{\partial u_{r}}{\partial r}\right|_{r=r_{w}}-i_{0}+\frac{k_{w}}{k_{h}} \frac{r_{w}}{2} \frac{\partial^{2} u_{w}}{\partial z^{2}}=0 .
$$

Equations (7), (8), (9), and (13) constitute the governing equations of consolidation by vertical drains incorporating the threshold gradient under the equal strain condition. The boundary conditions and initial conditions are as follows:

$$
\begin{aligned}
& \text { (1) }\left.\left(\partial u_{r} / \partial r\right)\right|_{r=r_{t}}=\gamma_{w} i_{0} \text {; } \\
& \text { (2) }\left.u_{w}\right|_{z=0}=0 \text {; } \\
& \text { (3) }\left.\left(\partial u_{w} / \partial z\right)\right|_{z=l}=0 \text {; } \\
& \text { (4) }\left.u_{r}\right|_{r=r_{w}}=u_{w} \text {; } \\
& \text { (5) }\left.\overline{u_{r}}\right|_{t=0}=u_{0} \text {; } \\
& \text { (6) } u_{r}\left(r_{t}, t\right)=u_{0} \text {. }
\end{aligned}
$$

Obviously, the governing equation describes a moving boundary problem which is addressed by a time-dependent parameter $r_{t}$. 
2.3. Solution of System. Taking the integral of (7) with respect to $r$ for both sides and introducing boundary condition (1) yields

$$
\frac{\partial u_{r}}{\partial r}=\frac{\gamma_{w}}{2 k_{h}}\left(\frac{r_{t}^{2}}{r}-r\right) \frac{\partial \varepsilon_{v}}{\partial t}+\gamma_{w} i_{0} \quad r_{w} \leq r \leq r_{t} .
$$

From (14), the following relationship can be obtained:

$$
\left.\frac{\partial u_{r}}{\partial r}\right|_{r=r_{w}}=\frac{\gamma_{w}}{2 k_{h}}\left(\frac{r_{t}^{2}}{r_{w}}-r_{w}\right) \frac{\partial \varepsilon_{v}}{\partial t}+\gamma_{w} i_{0}
$$

Substituting (15) into (13) yields

$$
\frac{\partial^{2} u_{w}}{\partial z^{2}}=-\frac{\gamma_{w}}{k_{w}}\left(\frac{r_{t}^{2}}{r_{w}^{2}}-1\right) \frac{\partial \varepsilon_{v}}{\partial t}
$$

Following Hansbo's derivation procedure [3], in which the variation of $\partial \varepsilon_{v} / \partial t$ with depth $z$ is ignored, (16) transforms into a second-order variable coefficient equation. Integrating (16) along the drain length at a given time and combining boundary conditions (2) and (3), the excess pore pressure in the drain can be derived as

$$
u_{w}=\frac{\gamma_{w}}{k_{w}}\left(\frac{r_{t}^{2}}{r_{w}^{2}}-1\right)\left(l z-\frac{1}{2} z^{2}\right) \frac{\partial \varepsilon_{v}}{\partial t} .
$$

By integrating (14) again and combining it with continuity condition (5), the excess pore pressure in the seepage zone can be derived as

$$
\begin{array}{r}
u_{r}=\frac{\gamma_{w}}{2 k_{h}}\left(r_{t}^{2} \ln \frac{r}{r_{w}}-\frac{r^{2}-r_{w}^{2}}{2}\right) \frac{\partial \varepsilon_{v}}{\partial t}+\gamma_{w} i_{0}\left(r-r_{w}\right)+u_{w} \\
r_{w} \leq r \leq r_{t} .
\end{array}
$$

Substituting the expression of $u_{r}$ into (4), the average excess pore pressure of the seepage zone can be expressed as

$$
\overline{u_{r t}}=\frac{\gamma_{w} r_{t}^{2}}{2 k_{h}} F_{a t} \frac{\partial \varepsilon_{v}}{\partial t}+\gamma_{w} r_{w} F_{b t} i_{0}+u_{w}
$$

where

$$
\begin{gathered}
F_{a t}=\frac{r_{t}^{2}}{r_{t}^{2}-r_{w}^{2}} \ln \frac{r_{t}}{r_{w}}+\frac{1}{4} \frac{r_{w}^{2}}{r_{t}^{2}}-\frac{3}{4} \\
F_{b t}=\frac{2 r_{t}^{2}}{3\left(r_{t} r_{w}+r_{w}^{2}\right)}-\frac{1}{3} .
\end{gathered}
$$

Setting $n_{t}=r_{t} / r_{w}$, the coefficients $F_{a t}$ and $F_{b t}$ can also be expressed as

$$
\begin{gathered}
F_{a t}=\frac{n_{t}^{2}}{n_{t}^{2}-1} \ln n_{t}+\frac{1}{4 n_{t}^{2}}-\frac{3}{4} \\
F_{b t}=\frac{2 n_{t}^{2}}{3\left(n_{t}+1\right)}-\frac{1}{3} .
\end{gathered}
$$

Substituting the expression of $u_{w}$ into (19) and using the relationship of (8), the following differential equation can be obtained:

$$
\frac{\partial \overline{u_{r t}}}{\partial t}=-\frac{2 c_{h}}{r_{t}^{2} \mu_{t}}\left(\overline{u_{r t}}-F_{b t} r_{w} \gamma_{w} i_{0}\right)
$$

in which

$$
\begin{gathered}
c_{h}=\frac{k_{h}}{\left(\gamma_{w} m_{v}\right)} ; \quad \mu_{t}=F_{a t}+\beta_{z}\left(\frac{n_{t}^{2}-1}{n_{t}^{2}}\right) ; \\
\beta_{z}=\frac{k_{h}}{k_{w}}\left(\frac{l}{r_{w}}\right)^{2}\left(\frac{2 z}{l_{t}}-\frac{z^{2}}{l_{t}^{2}}\right) ; \quad G=\frac{k_{h}}{k_{w}}\left(\frac{l}{d_{w}}\right)^{2},
\end{gathered}
$$

in which $G$ denotes well resistance.

A fully analytical solution for (22) is difficult since there is a time-dependent parameter $r_{t}$ in the expression. Xie et al. [20] demonstrated an approximate approach for onedimensional consolidation in consideration of the threshold gradient, certifying the reasonability of the solution through a comparative analysis with a numerical solution. Therefore, in this study, the approximate approach is applied similarly to Xie et al.s study [20] by assuming that $r_{t}$ is a constant for solving (22).

With the above assumption and according to the initial condition (5), the average excess pore pressure of the seepage zone can be obtained from (27) as

$$
\begin{array}{r}
\overline{u_{r t}}=\left(u_{0}-F_{b t} r_{w} \gamma_{w} i_{0}\right) e^{-8 T_{h} / \mu_{t}^{\prime}}+F_{b t} r_{w} \gamma_{w} i_{0} \\
r_{w} \leq r \leq r_{t}
\end{array}
$$

in which

$$
\mu_{t}^{\prime}=\frac{n_{t}^{2}}{n^{2}} \mu_{t} ; \quad T_{h}=\frac{c_{h}}{d_{e}^{2}} t
$$

The time factor $T_{h}$ is a dimensionless parameter for $t$. Furthermore, if $i_{0}$ approaches 0 , (24) will degenerate to Hansbo's solution [3].

Combining (8), (22), and (24), there is

$$
\frac{\partial \varepsilon_{v}}{\partial t}=\frac{2 k_{h}}{\gamma_{w} r_{t}^{2} \mu_{t}}\left(u_{0}-F_{b t} r_{w} \gamma_{w} i_{0}\right) e^{-8 T_{h} / \mu_{t}^{\prime}} \quad r_{w} \leq r \leq r_{t} .
$$

Substituting (24) and (26) into (18), then

$$
\begin{aligned}
& u_{r}= {\left[\ln \frac{r}{r_{w}}-\frac{r^{2}-r_{w}^{2}}{2 r_{t}^{2}}+\beta_{z}\left(\frac{n_{t}^{2}-1}{n_{t}^{2}}\right)\right] } \\
& \times \frac{\left(u_{0}-\alpha_{t} r_{w} \gamma_{w} i_{0}\right) e^{-8 T_{h} / \mu_{t}^{\prime}}}{\mu_{t}}+\left(\frac{r}{r_{w}}-1\right) r_{w} \gamma_{w} i_{0} \\
& r_{w} \leq r \leq r_{t} \\
& u_{w}=\frac{1}{\mu_{t}} \frac{n_{t}^{2}-1}{n_{t}^{2}} \beta_{z}\left(u_{0}-F_{b t} r_{w} \gamma_{w} i_{0}\right) e^{-8 T_{h} / \mu_{t}^{\prime}} .
\end{aligned}
$$


The excess pore pressure, $u_{r}$, should satisfy the boundary condition (6). Thus, for radius $r=r_{t}$, the following equation is obtained:

$$
\begin{aligned}
& {\left[\ln \frac{r_{t}}{r_{w}}-\frac{r_{t}^{2}-r_{w}^{2}}{2 r_{t}^{2}}+\beta_{z}\left(\frac{n_{t}^{2}-1}{n_{t}^{2}}\right)\right]} \\
& \quad \times \frac{\left(q_{0}-F_{b t} r_{w} \gamma_{w} i_{0}\right) e^{-8 T_{h} / \mu_{t}^{\prime}}}{\mu_{t}}+\left(\frac{r_{t}}{r_{w}}-1\right) r_{w} \gamma_{w} i_{0}=u_{0} .
\end{aligned}
$$

It can be rewritten as

$$
\frac{\lambda_{t}}{\mu_{t}}\left(R-F_{b t}\right) e^{-8 T_{h} / \mu_{t}^{\prime}}+\left(n_{t}-1\right)=R .
$$

$R$ is a dimensionless parameter for $i_{0}$.

\section{Consider}

$$
\lambda_{t}=\ln n_{t}+\left(\beta_{z}-\frac{1}{2}\right)\left(\frac{n_{t}^{2}-1}{n_{t}^{2}}\right) ; \quad R=\frac{u_{0}}{r_{w} \gamma_{w} i_{0}} .
$$

When $t$ approaches $\infty$, it can be deduced from (30) that

$$
n_{t}=R+1 \text {. }
$$

Parameters $n_{t}$ and $r_{t}$ can be determined from (30) if the time factor, $T_{h}$, is specified and vice versa. Furthermore, the average excess pore pressure, $\overline{u_{r t}}$, and the excess pore pressure, $u_{r}$, in seepage zone at any depth can be obtained from (24) and (29), respectively.

If $n_{t}=R+1 \geq n$, the moving boundary will reach the outer radius of the influence zone. Then, the correspondent time can be obtained from (30) by replacing $n_{t}$ with $n$. The average excess pore pressure, $\overline{u_{r t}}$, and the excess pore pressure, $u_{r}$, can be calculated by substituting $r_{t}=r_{e}$ into (24) and (27). On the other hand, if $R+1<n$, the moving boundary will never reach the outer radius of the influence zone during the whole consolidation process.

The average pore pressure of the whole zone includes the sweep zone and the nonsweep zone at a given depth $z$ and it can be defined as follows:

$$
\overline{u_{r}}=\frac{1}{\pi\left(r_{e}^{2}-r_{w}^{2}\right)} \int_{r_{w}}^{r_{e}} 2 \pi r u_{r} d r=\frac{n^{2}-n_{t}^{2}}{n^{2}-1} u_{0}+\frac{n_{t}^{2}-1}{n^{2}-1} \overline{u_{r t}} .
$$

Generally, the average degree of consolidation in terms of stress is defined as the ratio of the average effective stress to the average total stress. Thus, the degree of consolidation at a given depth, $U_{r}$, can be calculated by integrating the average excess pore pressure over the radius (from $r_{w}$ to $r_{e}$ ):

$$
U_{r}=\frac{1}{\pi\left(r_{e}^{2}-r_{w}^{2}\right)} \int_{r_{w}}^{r_{e}} \frac{u_{0}-u_{r}}{u_{0}} 2 \pi r d r=\frac{n_{t}^{2}-1}{n^{2}-1}\left(1-\frac{\overline{u_{r t}}}{u_{0}}\right) .
$$

The average degree of consolidation for the whole soil stratum based on the dissipation of excess pore pressure, $\overline{U_{r}}$, is then computed by integrating $U_{r}$ over the soil layer thickness:

$$
\overline{U_{r}}=\frac{1}{l} \int_{0}^{l} U_{r} d z=\frac{1}{l} \int_{0}^{l} \frac{n_{t}^{2}-1}{n^{2}-1}\left(1-\frac{\overline{u_{r t}}}{u_{0}}\right) d z .
$$

Note here that (35) can be computed through numerical integration. Therefore, an analytical solution system for the radial consolidation with the threshold gradient is obtained.

\section{Parametric Analysis}

This section conducts a parametric analysis of the consolidation results, that is, $u_{r}, U_{r}$, and $\overline{U_{r}}$, for the present solution. The dimensionless parameters $\xi=z / l$ and $T_{h}$ represent the depth $z$ and the time $t$, respectively. The parameter values are herein listed: $r_{e}=0.525 \mathrm{~m}, r_{w}=0.035 \mathrm{~m}, n=r_{e} / r_{w}=15$, $m_{v}=0.2 \mathrm{MPa}^{-1}, l=20 \mathrm{~m}, k_{h}=2.0 \times 10^{-8} \mathrm{~m} / \mathrm{s}$, and $k_{w}=1.0 \times 10^{-3} \mathrm{~m} / \mathrm{s}$.

The motions of the moving boundary predicted by (30) along with the various values of the dimensionless threshold gradients, $R$, are presented in Figure 4. In Figure 4(a), the restriction of the outer boundary of influence is disregarded (i.e., $n \rightarrow \infty$ ), while in Figure 4(b) $n$ is set as 25 . It can be drawn that (1) the flow gradually occurs from the vertical drain to the outside influence zone; (2) when $t \rightarrow \infty$, the value of $n_{t}$ approaches $R+1$, which confirms to a certain extent the validity of (32); (3) if $R+1<n$, the moving boundary will never reach the outer radius of the influence zone during the whole consolidation process; on the contrary, it will only reach the influence zone if $R+1 \geqslant n$; (4) regardless of the restriction of the outer boundary of the influence zone, the value of $n_{t}$ at $t=\infty$ would increase along with $R$; (5) as the value of $R$ increases, the time for the moving boundary to reach the outer boundary of the influence zone decreases under the condition that $R+1 \geqslant n$.

The motions of the moving boundary predicted at different depths for value of $R=10$ are presented in Figure 5. It can be observed that (1) when $t \rightarrow \infty$, the value of $n_{t}$ at different depths approaches the same value $n_{t}=R+1$; (2) the deeper the depth of $z$, the smaller the radius of the moving boundary at time $t$; and (3) with the increased value of $G$, the motion of the moving boundary decelerates.

When considering the threshold gradient, the average excess pore pressure is related to the motion of the moving boundary. Figure 6(a) shows the average excess pore pressure variations with varying depths; and Figure 6(b) shows the corresponding moving boundary. In the two figures, the dotted lines represent the results of $R=10$, while the solid lines represent the results of $R=20$. The comparison shows that (1) at the end of consolidation the average pore pressure will not completely dissipate, but it will maintain a long-term stable value; (2) the larger the value of $R$, the smaller the longterm average pore pressure; and (3) if $R+1<n$, the radial flow within the range from $R+1$ to $n$ does not occur during the consolidation process, which results in a slow dissipation rate in the pore pressure.

A further comparison of the average degree of consolidation $\left(\overline{U_{r}}\right)$ for various values of $R$ is presented in Figure 7. It can be seen from the figure that (1) in the early stage of consolidation the difference in the average degree of consolidation with a different $R$ is minuscule. With a consolidation time that has elapsed, the effect of $R$ on the degree of consolidation becomes obvious and (2) as $R$ increases, the 
TABLE 1: Parameters and values used in the case at SkaÊ-Edeby.

\begin{tabular}{ccccccc}
\hline$d_{e}(\mathrm{~m})$ & $d_{w}(\mathrm{~m})$ & $n=d_{e} / d_{w}$ & $C_{h}\left(\mathrm{~m}^{2} / \mathrm{y}\right)$ & $k_{h}(\mathrm{~m} / \mathrm{y})$ & $q_{w}\left(\mathrm{~m}^{3} / \mathrm{y}\right)$ & $l(\mathrm{~m})$ \\
\hline 1.575 & 0.18 & 8.75 & $0.45^{*}$ & 0.02 & 100 & 12 \\
\hline
\end{tabular}

${ }^{*}$ refers to the solution determined from back-calculation method by fitting the field data from Hansbo's study [14].



(a) Disregard the restriction of the outer boundary of influence

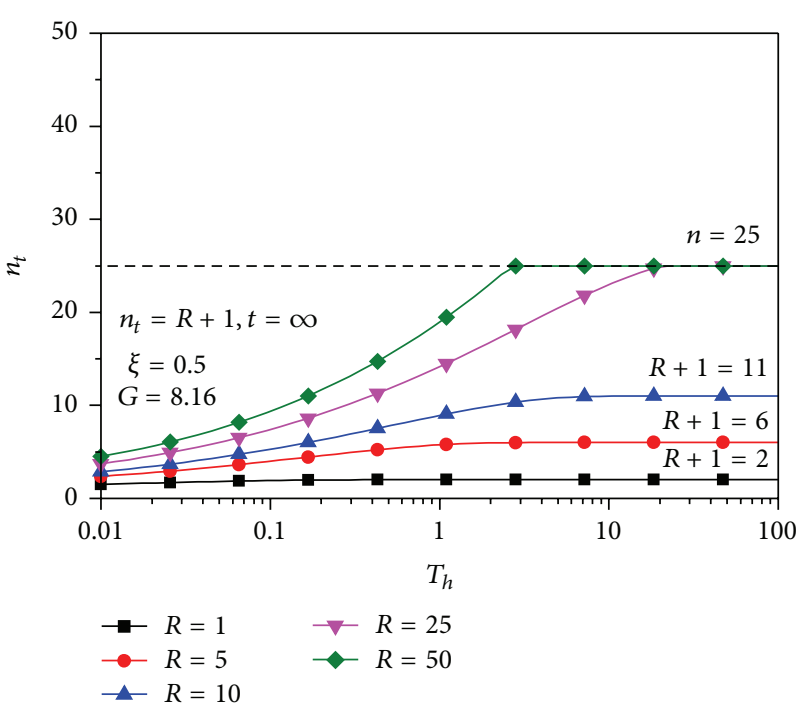

(b) Assume $n=25$

FIGURE 4: Comparison of the motion of the moving boundary for various $R$.

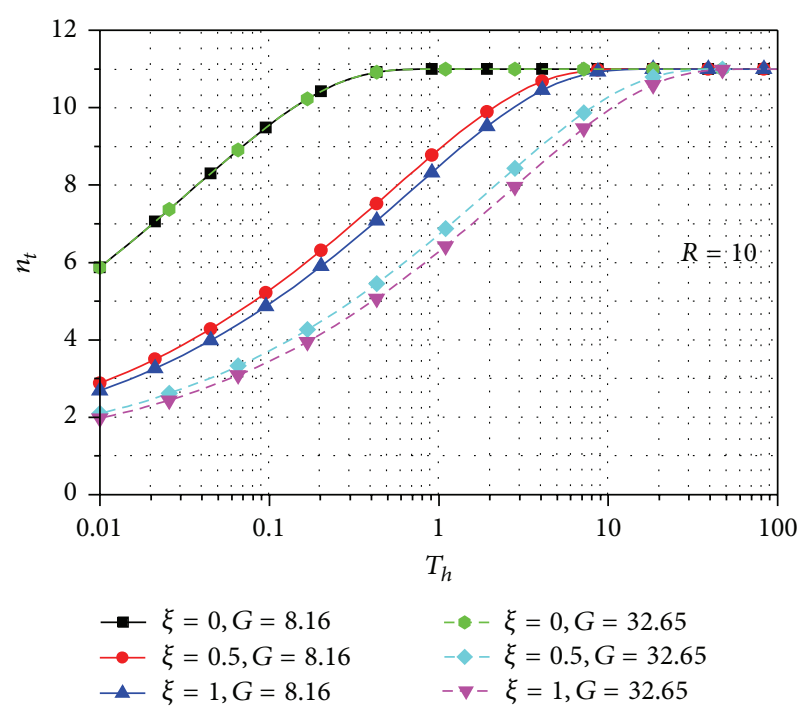

FIGURE 5: Comparison of the motion of moving boundary under different depths and $G(R=10)$.

development of consolidation accelerates and the average degree of consolidation, $\overline{U_{r}}$, increases.

\section{Application of Theory}

The field tests at SkaÊ-Edeby, located about $25 \mathrm{~km}$ west of Stockholm, are among the oldest and best-documented tests on the behavior of vertical drains throughout the world [14]. The details of this work and the results of the analysis were reported in Hansbo's papers [14]. The study included three test areas with sand drains, $0.18 \mathrm{~m}$ in diameter (test areas I III). The excess pore pressure between depths of 2.5 and $7.5 \mathrm{~m}$ in test areas II and III was selected for this study. The parameters used in the comparison are listed in Table 1. Except for the value of the consolidation coefficient, $C_{h}$, the other values are sampled from Hansbo's study [14]. Test area II and test area III were surcharged with an equivalent loading of $32 \mathrm{kPa}$ and $46 \mathrm{kPa}$. The radial average degree of consolidation, $U_{h}$, at a depth of $5 \mathrm{~m}$, as computed by the solutions of Hansbo [3] and the present paper, is plotted in Figure 8, respectively. The measured data are also plotted in this figure to aid comparative analysis. The consolidation degree calculated by the present solution with a consideration of the threshold gradient becomes related to the magnitude of the initial stress, which is not the case in Hansbo's solution [3] with a Darcian flow. It can be seen from the figure that the solutions both by Hansbo [3] $\left(C_{h}=\right.$ $\left.0.40 \mathrm{~m}^{2} / \mathrm{y}\right)$ and in the present paper $\left(C_{h}=0.45 \mathrm{~m}^{2} / \mathrm{y}\right.$ and $\left.i_{0}=0.365\right)$ confer with the measured data. Relatively, the newly present solution considering a threshold gradient for the flow gives a better agreement with the field measured value.

Another case involves a radial consolidation test that was conducted by Kim et al. [21], which was performed on a large block sample using a large consolidometer (diameter $=1.2 \mathrm{~m}$, height $=2.0 \mathrm{~m}$ ). The boundary for the consolidation test was 


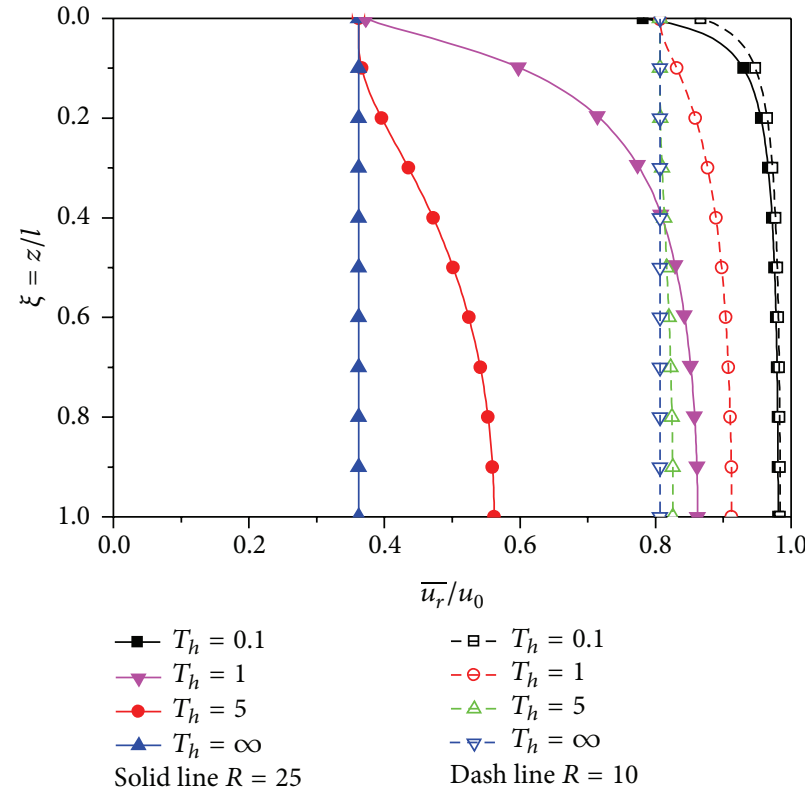

(a) The average EPP

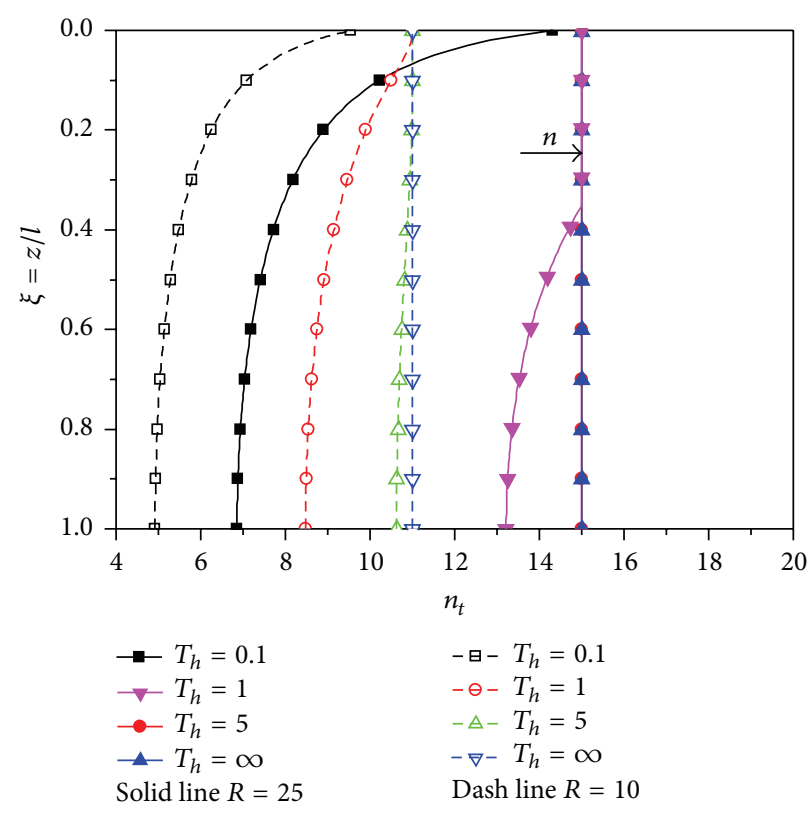

(b) The corresponding radius of moving boundary

FIgURE 6: Comparison of the average EPP and the corresponding radius of moving boundary at depth with $R=10$ and $R=25$.

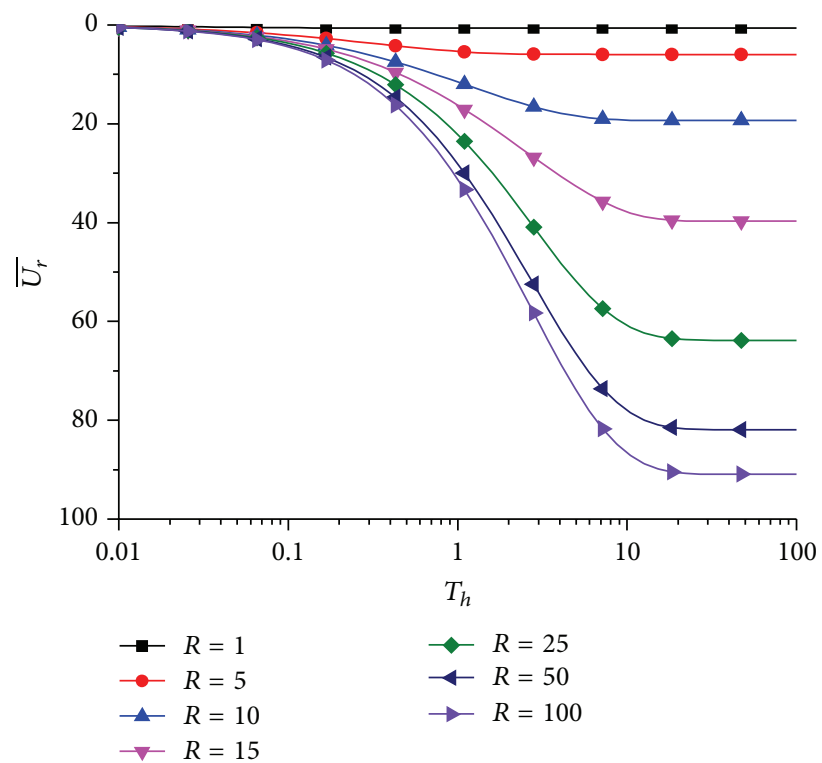

FIGURE 7: Comparison of the average radial degree of consolidation with times for different $R$.

particularly processed to ensure a solely radial consolidation condition. Finally, the block sample was consolidated under a vertical pressure of $300 \mathrm{kPa}$ for 3 weeks. The input parameters applied in the analysis are presented in Table 2. The parameters are summarized from Kim et al. [21] except the consolidation coefficient, $C_{h}$, which is determined using a back-calculation method.

The predictions from Hansbo's solution [3] under different values of $C_{h}$ and through the present solution are plotted

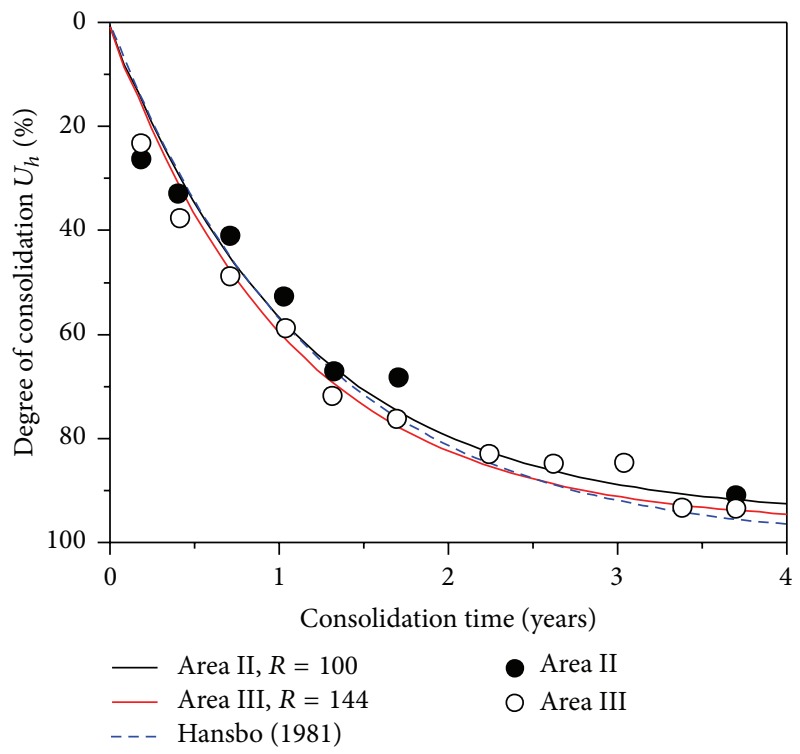

FIgURE 8: Comparison of consolidation curves and observed data at SkaÊ-Edeby.

in Figure 9, together with Kim et al.s [21] experimental data. It can be seen from the figure that all three predictions using Hansbo's solution (1981) deviate from the measured data with elapsed time. A better prediction of the consolidation process is obtained using the present solution with $C_{h}=1.39 \times$ $10^{-10} \mathrm{~m}^{2} / \mathrm{s}$ and $i_{0}=26.67$. Figure 9 indicates that the present solution, considering the threshold gradient, could be a better fit with the experimental results. 
TABLE 2: Parameters and values of Kim's radial consolidation test.

\begin{tabular}{lcccccc}
\hline$d_{e}(\mathrm{~m})$ & $d_{w}(\mathrm{~m})$ & $n=d_{e} / d_{w}$ & $C_{h}\left(\mathrm{~m}^{2} / \mathrm{s}\right)$ & $k_{h}(\mathrm{~m} / \mathrm{s})$ & $q_{w}\left(\mathrm{~m}^{3} / \mathrm{y}\right)$ & $l(\mathrm{~m})$ \\
\hline 0.60 & 0.05 & 12 & $1.39 \times 10^{-10^{*}}$ & $3.6 \times 10^{-10}$ & 5.0 & 2 \\
\hline
\end{tabular}

${ }^{*}$ refers to the solution determined from back-calculation method by fitting the experimental results from Kim et al.s study [21].

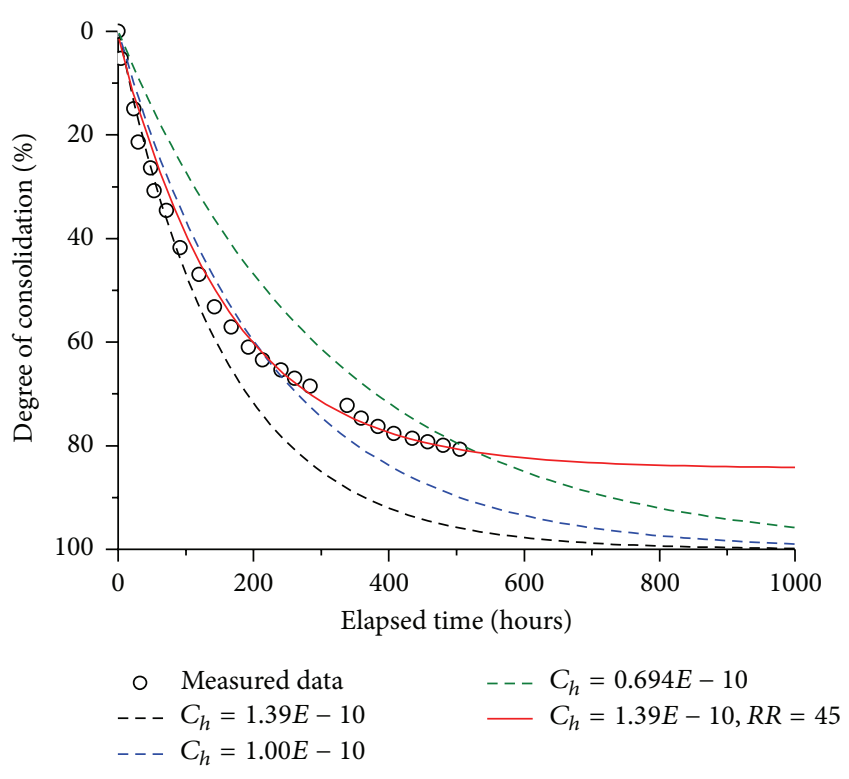

FIGURE 9: Comparison of theoretical results and observed data by Kim et al. [21].

\section{Conclusions}

Following Hansbo's approximate approach [3], the solution was developed for an equal strain consolidation theory of the vertical drain in consideration of the threshold gradient. Parametric analysis for the present solution was carried out in order to understand the effect of the threshold gradient on consolidation. The applicability of the present solution was demonstrated in two cases, wherein the comparisons with Hansbo's results and observed data were conducted. The following conclusions were obtained. (1) The flow with the threshold gradient does not occur throughout the whole unit cell instantaneously. It gradually occurs from the vertical drain to the outside. (2) The moving boundary would never reach the outer radius of influence if $R+1<n$; otherwise it will reach the outer radius of influence at some time. (3) The excess pore pressure will not completely dissipate, but it will maintain a long-term stable value at the end of consolidation. (4) The larger the threshold gradient, the larger the long-term excess pore pressure. (5) The present solution can predict the consolidation behavior in soft clay better than previous methods.

\section{Conflict of Interests}

The authors declare that there is no conflict of interests regarding the publication of this paper.

\section{Acknowledgment}

The support of this research by the National Natural Science Foundation of China (nos. 51179170 and 51308309) is gratefully acknowledged.

\section{References}

[1] R. A. Barron, "Consolidation of fine-grained soils by drain wells," Transactions of the American Society of Civil Engineers, vol. 113, pp. 718-742, 1948.

[2] H. Yoshikuni and H. Nakanodo, "Consolidation of soils by vertical drain wells with finite permeability," Soils Found, vol. 14, no. 2, pp. e35-e46, 1974.

[3] S. Hansbo, "Consolidation of fine-grained soils by prefabricated drains," in Proceedings of the 10th International Conference on Soil Mechanics and Foundation Engineering, A. A. Balkema, Ed., vol. 3, pp. 677-682, Stockholm, Sweden, 1981.

[4] A. Onoue, "Consolidation by vertical drains taking well resistance and smear into consideration," Soils and Foundations, vol. 28, no. 4, pp. 165-174, 1988.

[5] G. X. Zeng and K. H. Xie, "New development of the vertical drain theories," in Proceedings of the 12th International Conference on Soil Mechanics and Foundation Engineering, vol. 2, pp. 1435-1438, Rio de Janeiro, Brazil, 1989.

[6] C. J. Leo, "Equal strain consolidation by vertical drains," Journal of Geotechnical and Geoenvironmental Engineering, vol. 130, no. 3, pp. 316-327, 2004.

[7] C. Rujikiatkamjorn and B. Indraratna, "Design procedure for vertical drains considering a linear variation of lateral permeability within the smear zone," Canadian Geotechnical Journal, vol. 46, no. 3, pp. 270-280, 2009.

[8] C. Y. Ong, J. C. Chai, and T. Hino, "Degree of consolidation of clayey deposit with partially penetrating vertical drains," Geotextiles and Geomembranes, vol. 34, pp. 19-27, 2012.

[9] S. Artidteang, D. T. Bergado, J. Saowapakpiboon, N. Teerachaikulpanich, and A. Kumar, "Enhancement of efficiency of prefabricated vertical drains using surcharge, vacuum and heat preloading," Geosynthetics International, vol. 18, no. 1, pp. 35-47, 2011.

[10] B. Indraratna, C. Rujikiatkamjorn, J. Ameratunga, and P. Boyle, "Performance and prediction of vacuum combined surcharge consolidation at Port of Brisbane," Journal of Geotechnical and Geoenvironmental Engineering, vol. 137, no. 11, pp. 1009-1018, 2011.

[11] R. J. Miller and P. F. Low, "Threshold gradient for water flow in clay system," Proceedings of Soil Science Society of America, vol. 27, no. 6, pp. 605-609, 1963.

[12] J. Byerlee, "Friction, overpressure and fault normal compression," Geophysical Research Letters, vol. 17, no. 12, pp. 2109-2112, 1990.

[13] S. Hansbo, Consolidation of Clay with Special Reference to Influence of Vertical Sand Drains, vol. 18, Swedish Geotechnical Institute, 1960. 
[14] S. Hansbo, "Aspects of vertical drain design: Darcian or nonDarcian flow," Geotechnique, vol. 47, no. 5, pp. 983-992, 1997.

[15] H. W. Olsen, "Deviation from Darcys law in saturated clays," Proceedings of Soil Science Society of America, vol. 29, no. 2, pp. 135-140, 1965.

[16] J. K. Mitchell, Fundamentals of Soil Behaviour, John Wiley \& Sons, New York, NY, USA, 1976.

[17] G. Imai and Y.-X. Tang, "Constitutive equation of onedimensional consolidation derived from inter-connected tests," Soils and Foundations, vol. 32, no. 2, pp. 83-96, 1992.

[18] F. Tavenas, P. Leblond, P. Jean, and S. Leroueil, “The permeability of natural soft clays. Part I: methods of laboratory measurement," Canadian Geotechnical Journal, vol. 20, no. 4, pp. 629-644, 1983.

[19] F. Pascal, H. Pascal, and D. W. Murray, "Consolidation with threshold gradients," International Journal for Numerical and Analytical Methods in Geomechanics, vol. 5, no. 3, pp. 247-261, 1981.

[20] K.-H. Xie, K. Wang, Y.-L. Wang, and C.-X. Li, "Analytical solution for one-dimensional consolidation of clayey soils with a threshold gradient," Computers and Geotechnics, vol. 37, no. 4, pp. 487-493, 2010.

[21] R. Kim, S.-J. Hong, M.-J. Lee, and W. Lee, “Time dependent well resistance factor of PVD," Marine Georesources and Geotechnology, vol. 29, no. 2, pp. 131-144, 2011. 


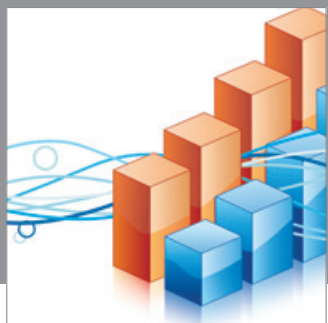

Advances in

Operations Research

mansans

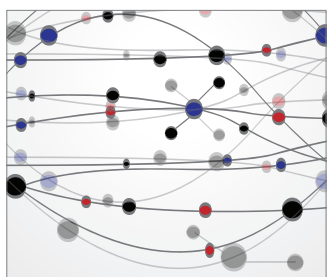

The Scientific World Journal

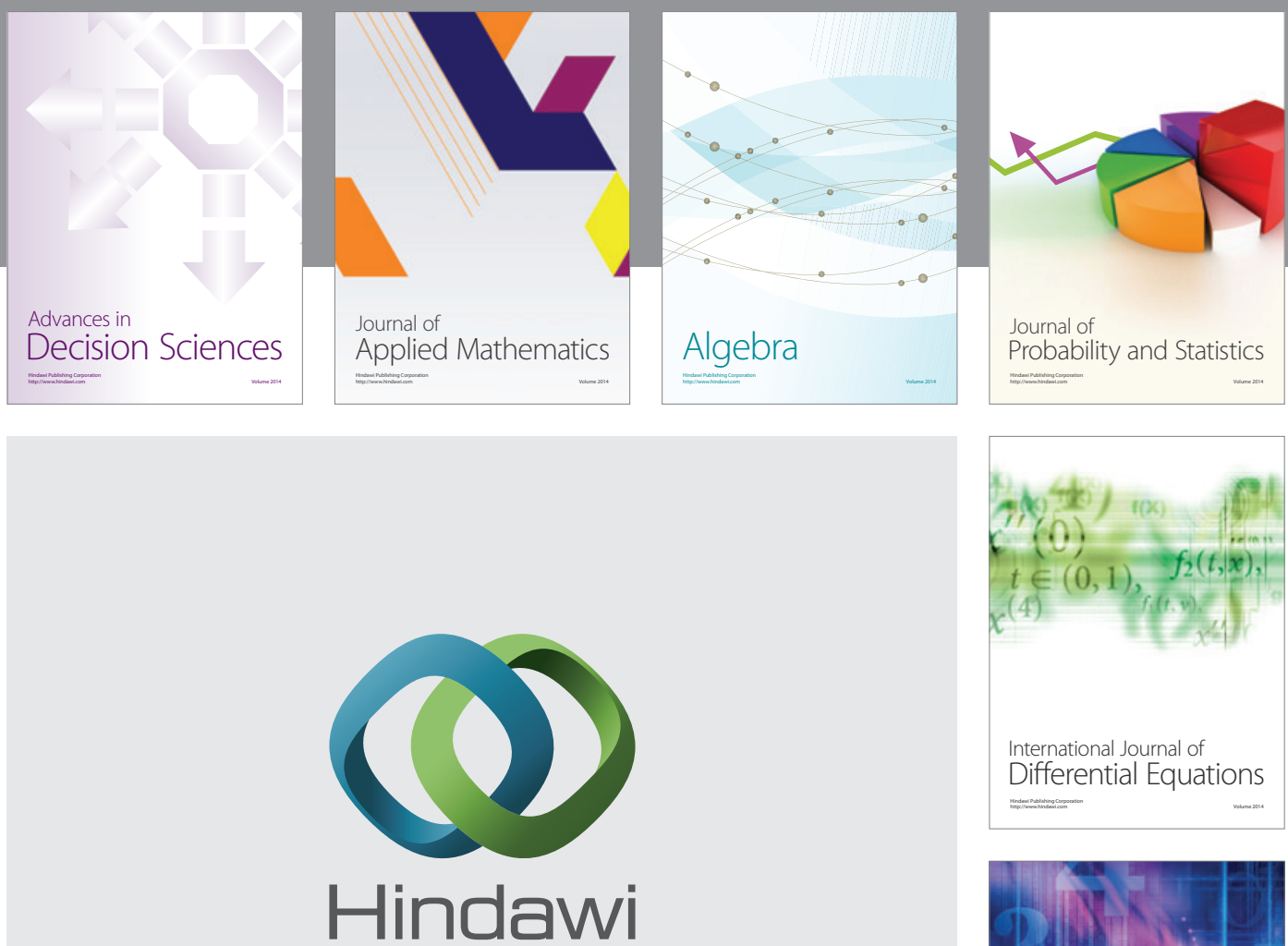

Submit your manuscripts at http://www.hindawi.com
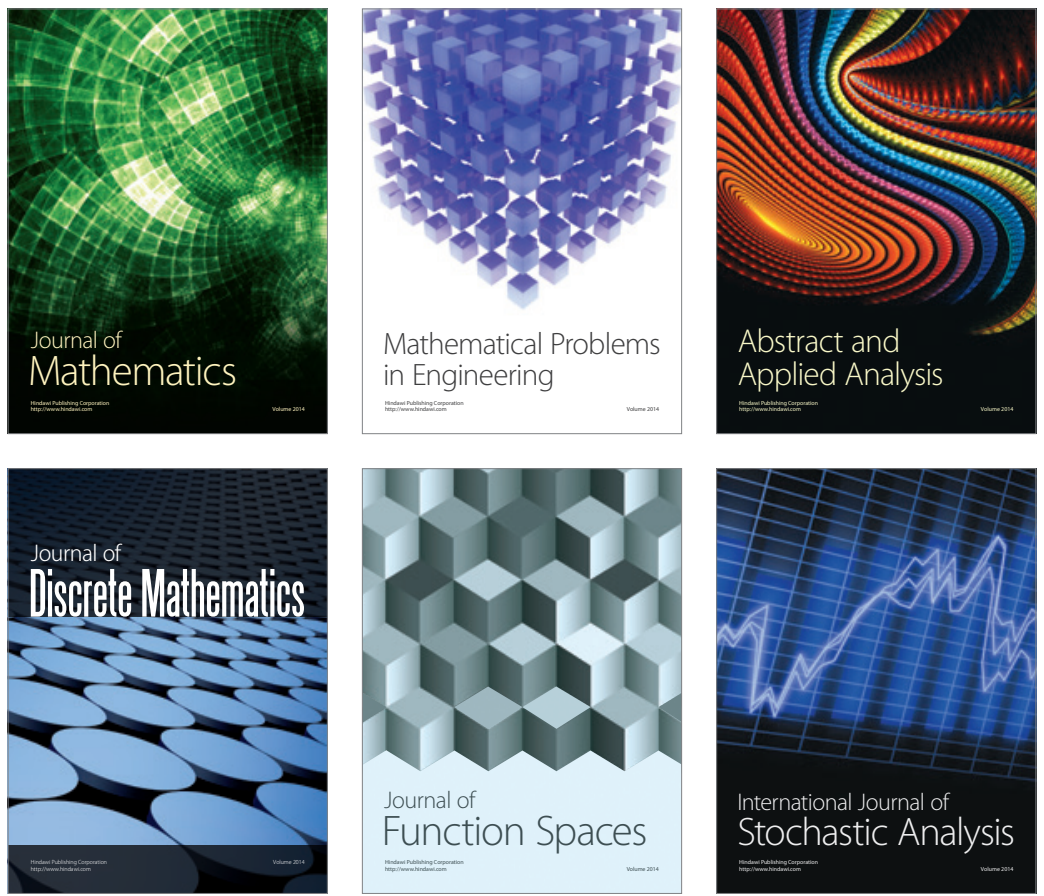

Journal of

Function Spaces

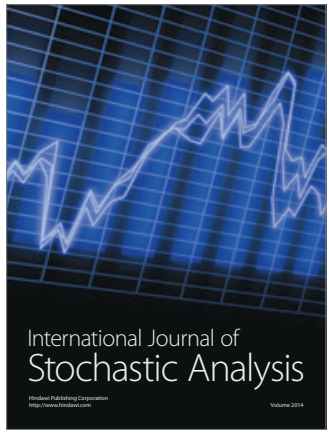

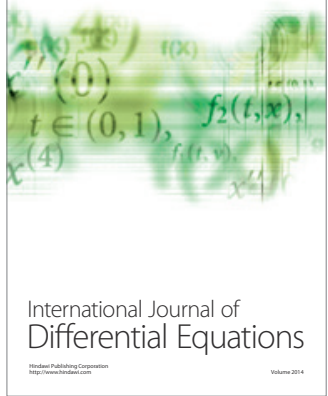
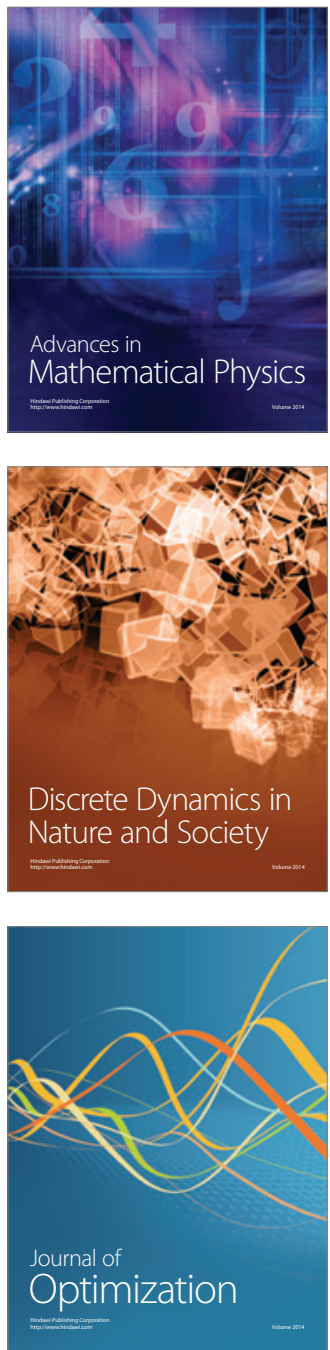